\title{
Prevención Cuaternaria: Ética Médica, Evaluación y Eficiencia en los Sistemas de Salud
}

\section{Quaternary Prevention: Medical Ethics, Evaluation and Efficiency in the Health Systems \\ Prevenção Quaternária: Ética Médica, Avaliação e Eficiência nos Sistemas de Saúde}

Miguel Pizzanelli. Grupo de interés en Prevención Cuaternaria de CIMF; Departamento de Medicina Familiar y Comunitaria de la Facultad de Medicina de la UdelaR. Uruguay. miguelpizzanelli@gmail.com (Autor correspondiente)

Marina Almenas. CIMF/WONCA; American Academy of Familiy Physicians. Puerto Rico. dra.almenas@gmail.com (Autora correspondiente) Robert Quirós. Universidad de Costa Rica; Área Salud de Moravia, San José, Costa Rica; Asociación de Especialistas en Medicina Familiar y Comunitaria. Costa Rica. rmqdoc@gmail.com

Carlos Pineda. Departamento de Medicina Familiar Universidad del Valle; Sociedad Colombiana de Medicina Familiar. Colombia.

capineda1@gmail.com

Esteban Cordero. Caja Costarricense de Seguro Social (CCSS). Costa Rica. estebch@gmail.com

Niurka Taureaux. Facultad de Ciencias Médicas "General Calixto García”. Cuba. niurka.toro@ infomed.sld.cu; niurkataureauxcu@gmail.com María de Lourdes Rojas. Universidad de Quintana Roo; Colegio Mexicano de Medicina Familiar. México. educación.rubi@gmail.com Karin Kopitowski. Departamento de Medicina y Directora del Departamento de Investigación del Instituto Universitario Hospital Italiano. Argentina. karin.kopitowki@gmail.com

Ricardo La Valle. UBA área Salud Publica; Instituto Universitario del Hospital Italiano. Argentina. lavayear@gmail.com

Mónica Nivelo. Departamento de Atención Primaria y Salud Familiar en la Universidad de Chile; Sociedad Chilena de Medicina Familiar.

Chile. monicla61@gmail.com

Verónica Menares. Hospital Clínico FUSAT; Sociedad Chilena de Medicina Familiar. Chile. v.menares।@gmail.com

Agustina Piñero. Facultad de Ciencias Médicas, Universidad Nacional de Córdoba. Argentina. agusep@ hotmail.com

Sonia Ortiz. Academia Médicos de Familia de PR. Puerto Rico. seofmd@aol.com

Rolando Herrera. Sociedad Boliviana de Medicina Familiar. Bolivia. enfoquealasalud@gmail.com

Alexis Correa Taja. Universidad de Ciencias Medicas (UCM). Nicaragua. acorrea5969 @ hotmail.com

\section{Colaboradores durante el Grupo de Trabajo de la Cumbre de Costa Rica}

Dr. Andrés Szwako, Paraguay. Dra. Carmen Nadal, Chile. Dr. Daniel Odio, Costa Rica. Dra. Diana Cáceres, Chile. Dr. Fernando Castañeda, Panamá. Dr. José Luis Contreras, Chile. Dra. Leticia Laura, México. Dra. María de Lourdes, México. Dra. Nina Monteiro, Portugal. Dr. Pedro Gómez, Costa Rica. Dra. Virginia Cardozo, Uruguay. Dra. Yamileth Quintero, Portugal

Cómo citar: Pizzanelli M, Almenas M, Quirós R, Pineda C, Cordero E, Taureaux N, et al. Prevención Cuaternaria: Ética Médica, Evaluación y Eficiencia en los Sistemas de Salud. Rev Bras Med Fam Comunidade. 2016;11(Suppl 2):75-85. http://dx.doi.org/10.5712/rbmfc11(0)1388
Financiación: ninguna declarada Aprobación ética: no aplicable. Conflicto de intereses: ninguna declarada. Procedencia y revisión por pares: revisado por pares. Recibido el: 15/08/2016.

Aceptado el: 15/09/2016 


\section{Resumen}

La prevención Cuaternaria consiste en desarrollar cuidados de salud y de ejercer la medicina, priorizando los cuidados centrados en la persona. Su marco conceptual se sustenta en aspectos éticos y filosóficos centrales en el ejercicio de la medicina, en aspectos epistemológicos y de carácter social. Su objeto es proteger fundamentalmente a los pacientes, pero también a los integrantes del equipo de salud, de los excesos de la medicalización y de los excesos o prácticas innecesarias o dañinas. El presente artículo es el resultado de la síntesis de los documentos de trabajo, discusión y propuestas llevadas adelante por un amplio grupo de comprometidos profesionales de CIMF, con interés particular sobre la Prevención Cuaternaria. Los materiales se produjeron en forma colaborativa por medio de un largo y complejo proceso de trabajo a distancia, realizado durante los foros preparatorios de la VI Cumbre Iberoamericana de Medicina Familiar y Comunitaria. A estos materiales se sumaron los documentos de trabajo elaborados durante la misma cumbre en San José de Costa Rica, en el mes de abril de 2016. El cometido de este artículo es difundir el estado de desarrollo y de compromiso actual con este enfoque y el destacado impulso que ha tenido en lberoamérica en los últimos cinco años. Por su relevancia, se pretende estimular una mayor difusión del concepto: la implementación de contenidos relacionados con él en la formación y en el nivel académico. A nivel político promover su consideración en la toma de decisión, en políticas de salud pública. Difundir a nivel poblacional y promover la elaboración de contenidos de calidad. Ofrecer pistas de reflexión y herramientas concretas para su aplicación.

\section{Abstract}

Quaternary Prevention as main focus and practice, promotes changes in developing health care and practicing medicine, prioritizing the person centred care. Their conceptual framework is sustained on ethical and philosophical aspects essential to medicine practice, in epistemological aspects of social and others related to political nature. Its objective is to protect fundamentally the patients, but also the members of the health group from excessive medicalization and unnecessary and hurtful practices. The present article is the result of the synthesis of the work documents, discussion and proposals carried forward by a large group of committed professionals from CIMF, with particular interest in Quaternary Prevention. The materials were produced in collaboration through a complex long distance work process, done during preparatory forums of the VI Ibero-American Summit of Family And Community Medicine. The work documents made during the same summit in San José of Costa Rica, in April 2016 were added to this material. The purpose of this document is to spread the state of development and current commitment to this approach and the outstanding initiative that it has had in Ibero America in the last five years. Due to its relevance, the intention is to stimulate greater dissemination of the concept; the implementation of content related to at in the training and academic levels. At a political level, to promote its consideration on decision making and public health issues so as to broadcast to demographic levels and promote the making of quality content. Finally to offer reflection clues to consider and concrete application tools.

\section{Resumo}

A Prevenção Quaternária consiste em desenvolver cuidados de saúde e exercer a medicina priorizando o cuidado centrado na pessoa. Seu arcabouço conceitual se sustenta em aspectos éticos e filosóficos fundamentais ao exercício da prática médica, também em aspectos epistemológicos e de caráter social. O seu objetivo é proteger os pacientes, mas também os membros da equipe de saúde, dos excessos da medicalização e das práticas excessivas ou desnecessárias ou prejudiciais. Este artigo é o resultado da síntese dos documentos de trabalho, discussão e propostas levada a cabo por um numeroso grupo de profissionais comprometidos com a CIMF, com particular interesse na Prevenção Quaternária. Os materiais foram produzidos de forma colaborativa através de um extenso e complexo processo de trabalho a distancia, realizado durante as reuniões preparatórias da $\mathrm{VI}$ Cúpula Ibero-Americana de Medicina de Família e Comunidade. A estes materiais se somaram os documentos de trabalho preparados durante a mesma cúpula em San Jose, Costa Rica, em abril de 2016. O objetivo deste artigo é o de difundir o estado de desenvolvimento e de compromisso atual com esta abordagem e o impulso notável que tem havido na Iberoamerica nos últimos cinco anos. Por sua relevância, pretende-se estimular uma maior difusão do conceito, bem como a implementação de conteúdos relacionados a ele na formação e em nível acadêmico; a nível político, promover sua consideração na tomada de decisões de políticas públicas de saúde; difundir a nível da população e promover o desenvolvimento de conteúdo de qualidade; oferecer pistas de reflexão e ferramentas práticas para sua implementação.
Palabras clave:

Prevención Cuaternaria

Relaciones médico paciente

Bioética

Medicalizacion

Sobre diagnóstico

Keywords:

Quaternary Prevention

Physician-Patient Relations

Bioethics

Medicalization

Overdiagnosis

Palavras-chave:

Prevenção Quaternária

Relações Médico-Paciente

Bioetica

Medicalização

Sobre diagnóstico 


\section{Introducción}

El presente artículo ha sido el resultado de un proceso de ajustada síntesis de los documentos de trabajo, discusión y propuestas de un amplio grupo de profesionales. Los materiales que condujeron a la elaboración de esta comunicación se produjeron en forma colaborativa por medio de un largo y complejo proceso de trabajo a distancia, que se realizara durante los foros preparatorios de la VI Cumbre Iberoamericana de Medicina Familiar y Comunitaria. A estos materiales se sumaron los documentos de trabajo elaborados durante la misma cumbre en San José de Costa Rica, en el mes de abril de 2016. Por tanto debe comprenderse que se trata de una versión muy concisa de toda la producción de contenidos realizada y que tiene por objetivo comunicar avances. Al documento completo puede accederse a través de las autoridades de las sociedades científicas miembros o tomando contacto con el Grupo de trabajo en Prevención Cuaternaria de la Confederación Iberoamericana de Medicina Familiar (CIMF).

En marzo de 2015 durante el 4을 Congreso Iberoamericano de Medicina Familiar y Comunitaria - la Prevención Cuaternaria fue por primera vez considerada como uno de los ejes temáticos - de un congreso Iberoamericano. Durante el mismo quedó formalmente constituido dentro de CIMF, el Grupo de interés en Prevención Cuaternaria. ${ }^{1}$ Asimismo es la primera vez que en una cumbre lberoamericana surge en la agenda como tema de trabajo el enfoque de Prevención Cuaternaria.

\section{Definición/Bases Conceptuales}

Se puede definir a la Prevención Cuaternaria como; "el conjunto de acciones implementadas para identificar a un paciente o a una población en riesgo de medicalización, protegerlos de intervenciones médicas invasivas y proponerles procedimientos y/o cuidados éticamente aceptables". ${ }^{2}$

La Prevención Cuaternaria como enfoque y práctica, promueve cambios en la forma desarrollar cuidados de salud y de ejercer la medicina, priorizando los cuidados centrados en la persona. Su marco conceptual se sustenta en aspectos éticos y filosóficos centrales en el ejercicio de la medicina. Su objeto es proteger fundamentalmente a los pacientes, pero también a los integrantes del equipo de salud, de los excesos de la medicalización y de los excesos o prácticas innecesarias o dañinas.

El concepto nació en 1986 cuando Marc Jamoulle combinó en un mismo esquema conceptual, a la línea del tiempo de la prevención, con la dimensión relacional (persona-médico): la visión de la persona cruzándose con la del médico respecto a un episodio de salud (Figura 1$).^{3}$

A partir del modelo propuesto por Leavell y Clark, ${ }^{4}$ se han establecido niveles de prevención. En cada nivel se emprenden acciones que pretenden evitar la aparición de la enfermedad, interrumpir su avance y mitigar sus secuelas cuando se han manifestado; tales medidas tienen una estrecha relación con las etapas de la historia natural de la enfermedad y se clasifican clásicamente hasta la irrupción de la prevención cuaternaria en tres niveles. De este modo la Prevención Cuaternaria no se ubica en forma lineal respecto a las anteriores. Puede aplicarse también en los campos de acción de los otros niveles clásicos, buscando que realicemos actividades necesarias, pertinentes, seguras, basadas en evidencia equilibrada y sin sesgos. El enfoque por tanto puede ser aplicado en todos los niveles tradicionales de prevención, pero no queda encerrado en ella. Se orienta a los cuidados de salud en general, por tanto no se reduce estrictamente a aspectos preventivos o curativos.

Podemos entender a la Prevención Cuaternaria como una estrategia que tiende a disminuir la iatrogenia a evitar intervenciones innecesarias y paliar los efectos adversos de las intervenciones necesarias. En otras palabras, sería una actualización y nueva conceptualización del "Primun non nocere" heredado por generaciones de médicos.

La Prevención Cuaternaria es más que una actividad objetiva. Permite reflexionar sobre las prácticas con amplia perspectiva desde diferentes niveles de complejidad. Esta reflexión y posterior modificación de prácticas puede aplicarse de modo diverso y amplio como por ejemplo: reflexionar sobre la manera de pensar la organización de los servicios de salud, el modo de encarar la comunicación con las personas, el enfoque de las preguntas de investigación, el balance riesgo beneficio de los cribados, el efecto del sobrediagnóstico y el sobretratamiento, las consecuencias de los tratamientos 


\begin{tabular}{|c|c|c|}
\hline \multirow{2}{*}{$\begin{array}{l}\text { conciencia o } \\
\text { sensación del } \\
\text { paciente } \\
\text { sensación de } \\
\text { salud }\end{array}$} & \multicolumn{2}{|c|}{$\begin{array}{l}\text { Conocimiento científico médico } \\
\text { evolución natural de la enfermedad }\end{array}$} \\
\hline & $\begin{array}{l}\text { I (Prevención) Primaria } \\
\text { Medidas adoptadas para evitar o } \\
\text { eliminar la causa de un problema de } \\
\text { salud en un individuo o población } \\
\text { antes de que se presente. Incluye la } \\
\text { promoción de la salud y la protección } \\
\text { específica (por ejemplo, la } \\
\text { inmunización). }\end{array}$ & $\begin{array}{l}\text { II (Prevención) Secundaria } \\
\text { Medidas adoptadas para detectar un } \\
\text { problema de salud en una etapa } \\
\text { temprana, en un individuo o una } \\
\text { población, lo que facilita la curación o la } \\
\text { reducción o la prevención de su } \\
\text { propagación o efectos a largo plazo (por } \\
\text { ejemplo: métodos, cribados, detección } \\
\text { de casos y diagnóstico precoz). }\end{array}$ \\
\hline $\begin{array}{l}\text { sensación de } \\
\text { enfermedad }\end{array}$ & $\begin{array}{l}\text { IV (Prevención) Cuaternaria } \\
\text { Medidas adoptadas para identificar } \\
\text { a un paciente en riesgo de } \\
\text { medicalización excesiva, para } \\
\text { protegerlo de intrusiones médicas } \\
\text { adicionales, y sugerir intervenciones } \\
\text { éticamente aceptables. }\end{array}$ & $\begin{array}{l}\text { III (Prevención) Terciaria } \\
\text { Medidas adoptadas para reducir los } \\
\text { efectos de los problemas crônicos de } \\
\text { salud de un individuo o población para } \\
\text { reducir al mínimo el deterioro funcional } \\
\text { debido a un problema de salud agudo o } \\
\text { crônico (por ejemplo la prevención de } \\
\text { complicaciones de la diabetes). Incluye } \\
\text { la rehabilitación. }\end{array}$ \\
\hline
\end{tabular}

Figura 1. Las definiciones ya publicadas de Prevención I, II y III son complementadas por la Prevención IV y ofrecen una nueva visón de los campos de actividad del medico familiar.

Fuente: adaptado de Jamoulle M, Gomes LF. Prevenção Quaternária e limites em medicina. Rev Bras Med Fam Comunidade. 2014;9(31):186-91. Disponível em: http://dx.doi.org/10.5712/rbmfc9(31)867

innecesarios, el conflicto de intereses. Los excesos en los cuidados y el agotamiento de recursos que producen, admiten un análisis desde la ética, ya pueden provocar que existan personas con cuidados insuficientes o población con dificultades para acceder a atención adecuada, un hecho bastante frecuente en la realidad de Iberoamérica. ${ }^{5}$

\section{Medicalización}

Se entiende por Medicalización al proceso de convertir situaciones vitales, en cuadros patológicos y pretender resolver, mediante la Medicina, situaciones que no son médicas, sino sociales, profesionales o de las relaciones interpersonales. ${ }^{6,7}$

También es la pretensión de la Medicina resolver problemas que no son médicos, o al menos no eran considerados anteriormente. Los profesionales sanitarios son, a la vez, actores y víctimas de dicho proceso. ${ }^{8,9}$ La medicalización tiene como principales consecuencias la transformación de personas sanas en enfermos, el aumento de daños iatrogénicos, el consumo de recursos sanitarios y la falta de procesos encaminados a regular las expectativas de la población, delimitar el campo de acción de la medicina, fomentar el autocuidado y actuar de acuerdo con las evidencias sanitarias existentes.

\section{Descripción de la situación actual sobre la difusión y aplicación en Iberoamérica}

A pesar de existir un importante impulso para dar a conocer el concepto desde las sociedades científicas integrantes de la $\mathrm{CIMF}^{10}$ se reconoce la necesidad de mejorar la difusión así como el impacto del mismo.

\section{Metodo}

Para disponer de datos más fieles sobre la realidad, se realizó una encuesta entre médicos participantes del grupo de trabajo, sobre algunos temas relacionados a la difusión y aplicación de la Prevención Cuaternaria en cada país, sistema de salud y gobiernos. 
Se recolectaron datos a través de encuestas sobre la situación en trece países: Argentina, Bolivia, Chile, Colombia, Costa Rica, Cuba, España, México, Nicaragua, Paraguay, Puerto Rico, República Dominicana y Uruguay.

Del total de los encuestados, un 58\%, afirmaron que se ha promovido la incorporación de la Prevención Cuaternaria en sus países. Solo dos países afirmaron que se hayan logrado adoptar recomendaciones basadas en los principios de la Prevención Cuaternaria en la práctica de los médicos familiares.

\section{Resultados}

Un 58\% reportó conocimiento sobre la realización de actividades con la temática en los congresos o eventos científicos o formativos de sus sociedades.

Un 50\% reportó espacios de reflexión sobre el tema. Sin embargo, sólo cuatro países (un 33\%) reportaron publicaciones relacionadas al tema. Tres países comunicaron conocimiento de proyectos de investigación con un enfoque relacionado.

Sólo dos de las encuestas se reportó que hubiera conocimiento sobre Prevención Cuaternaria en la población general.

Dos terceras partes de los encuestados afirmaron abordar temas de Prevención Cuaternaria con los pacientes. Dos países (16\%) reportaron conocimiento de la Prevención Cuaternaria por parte de instituciones gubernamentales en su país. Sólo cuatro países (33\%) afirmaron que hubiera acciones de Prevención Cuaternaria por parte el gobierno de su país. Siete países, un 63\%, reconocieron la posibilidad real de que la Prevención Cuaternaria sea tema de discusión a nivel gubernamental.

\section{Discusion}

Si bien los resultados obtenidos, no pueden considerarse totalmente representativos de la realidad latinoamericana, la difusión a nivel de la población, en el ámbito médico y académico, así como entre las autoridades sanitarias y tomadores de decisiones sigue siendo necesaria y se considera insuficiente.

La metodología de trabajo para la elaboración de los documentos preparatorios y de los documentos orientados a elaborar las recomendaciones para la cumbre, fue participativa y se desarrolló en dos etapas: una virtual y otra presencial. La etapa virtual fue a través de redes, con trabajo y comunicación a distancia y tuvo lugar entre agosto de 2015 hasta abril de 2016. En la etapa presencial durante la misma cumbre, se elaboraron: las conclusiones finales, recomendaciones, aportes de síntesis para la declaración final y se presentaron conclusiones y productos de comunicación en plenario.

Se desarrollaron contenidos y líneas estratégicas en tres ejes de trabajo: recomendaciones para introducir el concepto en la formación de los médicos de familia (pregrado y posgrado), en la comunidad, en la población general y a nivel de las políticas públicas de salud.

\section{Líneas de Acción en el Escenario Académico}

A nivel académico, es necesario difundir, consolidar y aplicar el concepto de Prevención Cuaternaria entre los profesionales de la salud, en particular en el currículo de pregrado y postgrado de las universidades.

Se considera prioritario promover el concepto de Prevención Cuaternaria y plantearlo conceptualmente como una actividad clínica genuina en nuestra práctica comunitaria diaria. Profundizar y promover reflexión entre colegas, sobre su sentido y el modo de aplicación en situaciones concretas.

Vinculación con las Universidades para realizar propuesta de inclusión de Prevención Cuaternaria en el currículo.

La universidad debe tomar como propios los problemas identificados en el entorno y contribuir a su solución y así ganará prestigio ante la comunidad, quien irá transformando su opinión sobre la institución y esta jugará también el rol que le corresponde sobre todo en lo concerniente a la educación y formación de pregrado y posgrado. ${ }^{11-15}$ 


\section{Estrategias para la gestión curricular del concepto de Prevención Cuaternaria en la formación de los profesionales de la salud de lberoamérica}

Elaborar documento de recomendaciones curriculares con la conceptualización básica de la Prevención Cuaternaria y su implicación en la salud, planteando las competencias que implica con sus respectivos saberes cognitivos, procedimentales y actitudinales.

1. Educación Formativa: pregrado y posgrado Insertar módulo de Prevención Cuaternaria en pregrado y posgrado.

Promover la participación en la formación docente en Prevención Cuaternaria.

Favorecer la utilización de herramientas de lectura crítica de artículos que permita desarrollar pensamiento crítico y análisis de sesgos. Desarrollar talleres de discusión de dilemas éticos, comunicación efectiva, cuidados centrados en la persona, decisiones compartidas. Favorecer el desarrollo de investigación con focalidad en temas de interés de la Prevención Cuaternaria en médicos residentes. Inclusión de Prevención Cuaternaria en exámenes profesionales y examen de Certificación por los Consejos de las especialidades.

2. Generar un acervo documental en Prevención Cuaternaria.

Desarrollar un Observatorio en Prevención Cuaternaria.

Generar una normoteca digital sobre Prevención Cuaternaria.

Desarrollar boletines, videos, de Prevención Cuaternaria.

Favorecer el trabajo interdisciplinario en Prevención Cuaternaria (equipo de salud, otros profesionales y técnicos sanitarios, especialistas focales).

Los temas de interés de la Prevención Cuaternaria incluyen:

- Medicalización;

- Concepto de salud y enfermedad;

- Sobrediagnóstico; ${ }^{16}$

- Exceso en intervenciones preventivas; ${ }^{17}$

- Cribado de cáncer; $;^{18}$

- Riesgo cardiovascular;

- Chequeos ejecutivos;

- Efectividad y seguridad de la vacunación;

- Mercadeo de enfermedades;

- Excesos en uso de paraclínicos, Imágenes diagnósticas e incidentalomas;

- Sobremedicalización: polifarmacia, deprescripción, uso racional de medicamentos, eventos adversos y efecto cascada farmacológica;

- $\quad$ Seguridad del paciente;

- Estudio de los factores que afectan la medicalización, Medicina basada en la evidencia: Ética de la investigación, la Bioética en la práctica clínica actual;

- Método clínico: comunicación efectiva, método clínico centrado en la persona, método clínico centrado en la relación, cuidados centrados en la persona, toma de decisiones compartidas y ayudas de decisión.

\section{Ruta curricular}

El proceso de cómo concretar todo lo anterior en un micro-currículo, es una tarea compleja, en la medida que cada universidad tiene su propio currículo. El ejercicio que se hará a continuación es un mero acercamiento teórico a contenidos que podrían incluirse en determinados cursos en una determinada secuenciación curricular.

\section{Propuestas para el establecimiento de políticas acordes con el pensamiento de Prevención Cuaternaria}

Toda reforma de un sistema de salud debe ser iniciada desde una perspectiva ética y debe estar fundamentada en objetivos de desarrollo sostenible, con principios políticos claramente formulados, que asegure el cuidado 
ecológico, la participación de la comunidad y los distintos sectores en su conjunto para una co-creación de soluciones para un cambio sustentable. ${ }^{19}$

Una estrategia integral de desarrollo humano y salud, con enfoque de equidad y con impacto en los determinantes de salud requiere: un sistema de salud que persiga la superación de la pobreza y el hambre; asegure atención de salud y promueva el bienestar para todos a lo largo del ciclo vital; aporte a la educación y oportunidades de desarrollo en todo el curso de vida; logre equidad de género; contribuya a la disponibilidad y al manejo sostenible de agua y servicio sanitario; impulse el acceso a energía confiable y sustentable; aporte al crecimiento económico sostenible con trabajo digno; colabore en el diseño de una infraestructura resiliente a los desastres; fomente la innovación y la industrialización sustentable; impulse la reducción de la inequidad entre países; aporte al fomento de patrones de producción y consumo sostenibles; asuma medidas para para combatir el cambio climático y sus impactos; impulse el uso racional de los recursos naturales, para un desarrollo sostenible; colabore en la protección del ecosistema terrestre y la biodiversidad; participe en la promoción de la paz en ciudades inclusivas, fortalezca los medios para implementar la asociación global, en este caso la salud global, para un desarrollo sustentable.

En este contexto el enfoque de prevención cuaternaria tiene un aporte esencial. Si la consideramos como movimiento, ${ }^{20}$ puede impulsar la promoción de cambios en la forma de ser y ejercer la medicina y en el modo de organizar los sistemas de salud con énfasis en los aspectos éticos y filosóficos con el objeto de proteger a los pacientes y a los integrantes del equipo de salud de los excesos de la medicalización y de la mercantilización y afán de lucro de algunos actores. Para iniciar este cambio se proponen los siguientes principios:

1. La salud es un derecho y no un objeto de mercado. ${ }^{21}$

2. La salud no puede ser comprendida analizando solamente el sector salud y prescindiendo de su contexto, es necesario incorporar la mirada social y política al ejercicio de la profesión para incluir los determinantes sociales en el desarrollo de políticas de salud y en el ejercicio mismo de la profesión. El modelo médico predominante en la actualidad, analizado desde el punto de vista sociológico, ha sido caracterizado como hegemónico, reduccionista, deshumanizado, fragmentado y mercantilista. ${ }^{22}$

3. La Medicina como ciencia es predominantemente social. La Medicina debe abandonar el enfoque reduccionista del positivismo y neopositivismo y asumirse como arte antes que como ciencia para recuperar la capacidad de atender personas que sienten y que sufren sin que esto signifique no valorar ni incorporar todos los conocimientos que la ciencia y tecnología aporten ni descuidar el conocimiento biomédico.

4. La definición de un sistema de salud es política y forma parte indisoluble de la definición del modelo de país al que pertenece.

5. El ejercicio de la Medicina está atravesado y determinado por la política y los médicos, en consecuencia, no deben ser actores pasivos, deben aportar su visión particular de la sociedad y participar en la definición del modelo a seguir.

6. La Atención Primaria de Salud (APS) sólo funcionará estando integrada a un sistema de salud. La APS no puede funcionar en forma aislada del resto del Sistema de salud. Debe estar integrada a todos los niveles de atención distribuida transversalmente en los todos los niveles de complejidad.

7. La medicina familiar debe plantearse como una especialidad transversal.

8. La complejidad en medicina debe ser definida en función de la capacidad de resolución de problemas de los agentes de salud y no en función de la tecnología.

9. Los conocimientos y las medicinas son bienes sociales. El séptimo principio consagrado por el preámbulo de la Constitución de la Organización Mundial de la Salud, "La extensión a todos los pueblos de los beneficios de los conocimientos médicos, psicológicos y afines es esencial para alcanzar el más alto grado de salud". ${ }^{23}$ La existencia de barreras a su acceso, patentes y costos excesivos atentan contra la tarea de alcanzar esa meta.

Se mencionan algunas formas concretas de conducir el trabajo en el nivel político:

- Identificar políticas públicas de los países firmantes de la Carta de San José, que no cumplan los criterios mencionados en ella. 
- Crear espacios de trabajo en cada sociedad científica, con el objetivo de revisar y actualizar periódicamente las directrices nacionales implementadas.

- Promover la reflexión y sugerir el diseño de políticas asociadas a la divulgación de la información relacionada a intervenciones sanitarias.

\section{Propuesta de líneas de acción para difundir y aplicar el concepto de Prevención Cuaternaria en la población general}

La medicalización y la gestión del riesgo como práctica sanitaria, provoca la llegada de categorías desconocidas anteriormente en la medicina. "nuevos enfermos" o "enfermos-sanos". A personas sanas se les otorga nacionalidad en el mundo de los enfermos, muchas veces, con mucho para perder en términos de daños y nada para ganar en términos de salud. Existen muchos motores que han conducido a esta situación "en la cual casi nadie está sano". Uno de esos motores es la preocupación desmedida por parte del público en general por mantener salud a precios muy altos. Los pacientes, muchas veces, impulsan la medicalización, ${ }^{24}$ ajenos a los daños a que esto puede exponerlos. Suele estar presente la demanda "de la cura" y la abolición de todo síntoma. A veces el paciente no asocia que eso que le pasa y que se expresa por un síntoma, tenga que ver con "cosas de la vida" y no con enfermedades. En algunos sectores, la salud es vista como un bien de consumo y no como un derecho. Esta situación favorece la medicalización, la profusión de estudios diagnósticos muchas veces innecesarios y dañinos y la prescripción de fármacos. En ocasiones, la sociedad ha desplazado al campo médico, la búsqueda de soluciones inherentes a la realidad subjetiva y social de las personas y la obsesión por la salud perfecta se ha convertido en un factor patógeno.

Existe un fenómeno denominado paradoja de la salud que se observa esencialmente en países desarrollados y en las capas sociales medias y altas de los países en desarrollo. Esta paradoja consiste en que a mayor salud objetiva (indicadores de salud, expectativa de vida) más son los problemas de salud declarados y que cuanto mayores son los recursos empleados en salud, hay mayor número de personas que se sienten enfermas. Es decir, que la sociedad vive de manera insana por la preocupación exagerada por la salud. Suele existir tal dependencia de la Medicina "ante cualquier duda consulte a su médico" que se incapacita a las personas para autocuidarse, tolerar el sufrimiento y el malestar.

Se propone avanzar hacia un modelo de trasmisión de información, en todos los soportes existentes o posibles: panfletos en papel, folletería, cartelería, avisos radiales, televisivos, redes sociales, páginas web, blogs, videos. La finalidad de los materiales de difusión es presentar contenidos en forma balanceada, con todos los pros y contras, garantizar contenidos de calidad, que eviten sesgos. De este modo cada persona, tendrá una oportunidad mas equilibrada de elegir de acuerdo con sus valores y ejercer su derecho a la autodeterminación.

Propuestas:

- Difundir el concepto de Prevención Cuaternaria como una dimensión funtamental en la práctica médica actual.

- Promover que las intervenciones deben establecerse según las necesidades de la persona, según niveles de prevención y basado en la mejor evidencia disponible.

- Establecer claramente con evidencia científica cuán adecuado es un examen según su rendimiento y costo/efectividad en cada nivel de prevención según las necesidades de cada persona, familia o comunidad.

- Establecer mensajes que estimulen el diálogo significativo entre el público y sus médicos de familia.

- Recomendar el uso de mensajes que no conduzcan a la medicalización en las etapas normales de la vida y en los problemas existenciales.

- Establecer prácticas en las que las intervenciones sean pertinentes desde el punto de vista ético-científico y centrado en las personas, basadas en decisiones compartidas.

- Favorecer mensajes que estimulen a los pacientes a preguntar, conocer sus opciones los pros y contras de cada una de ellas. 
- Promocionar entre los médicos, la población general, políticos y los tomadores de decisiones de salud, la búsqueda en aquellos portales o bibliotecas virtuales la documentación necesaria para acentuar sus bases de conocimientos en Prevención Cuaternaria, tales como; Choosing Wisely, USPSTF, Evalmed.es.

- Promover la utilización de los documentos, carteles, folletos, y videos educativos a los que se puede acceder a través de las redes de Prevención Cuaternaria de CIMF.

\section{Conclusiones}

A modo de conclusiones se incluyen las propuestas priorizadas para el documento final de la cumbre:

- Difundir el concepto de Prevención Cuaternaria como enfoque fundamental en la práctica de las profesiones de la salud, en el pregrado y postgrado, en la formación continua, en la investigación, elaborando un documento de recomendaciones para ello y estableciendo contacto con las diferentes organizaciones que definen las políticas de Educación-médica actual.

- Promover la no medicalización de los acontecimientos propios de las etapas de la vida, a través de estrategias elaboradas junto a los equipos de salud y la comunidad.

- Incentivar que las intervenciones sanitarias dirigidas a la población, estén basadas en la mejor evidencia científica y sean éticamente aceptables para el contexto local y estén centradas en las personas.

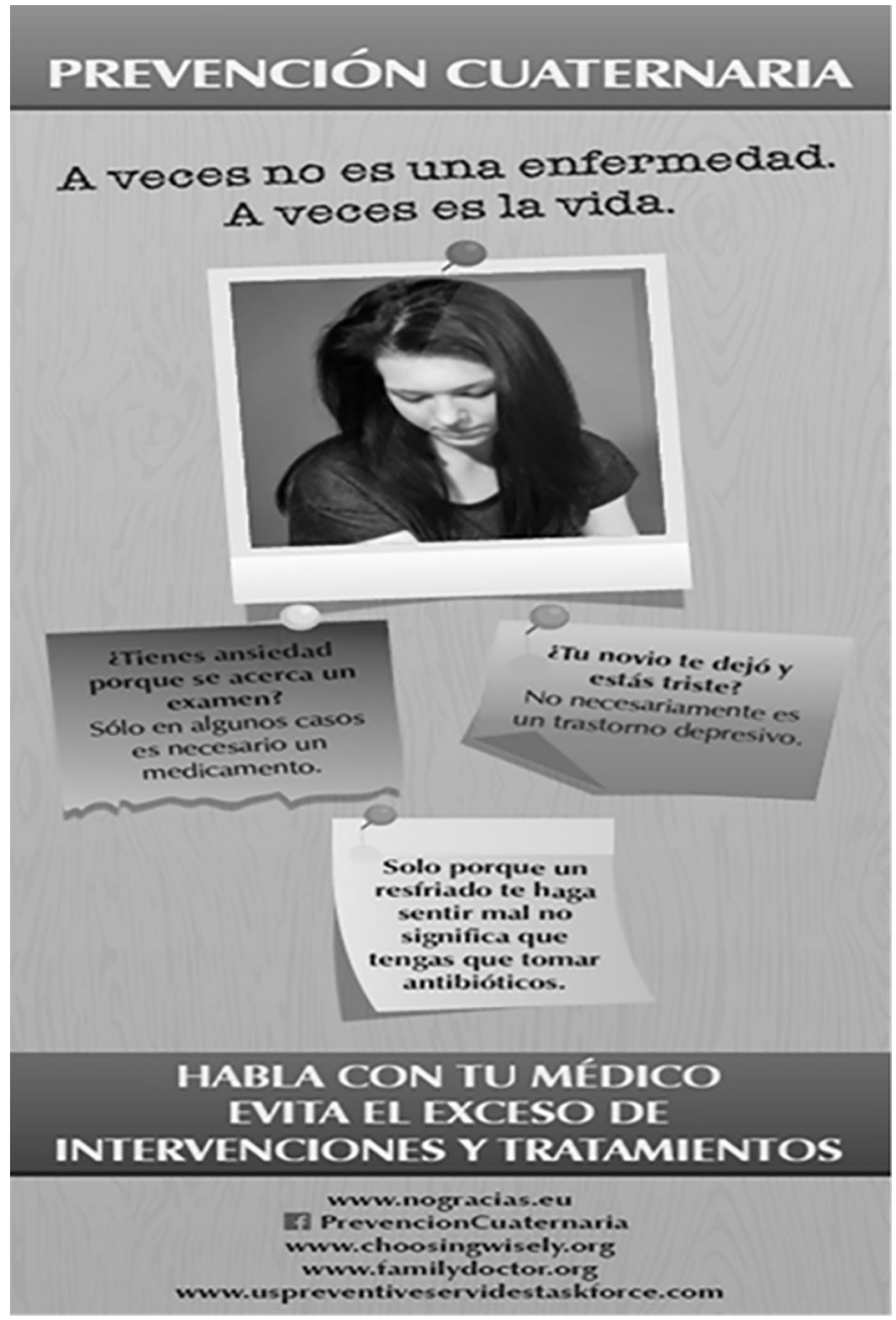

Prevención cuaternaria se refiere a las actividades que atenúan o evitan las intervenciones innecesarias o excesivas. 

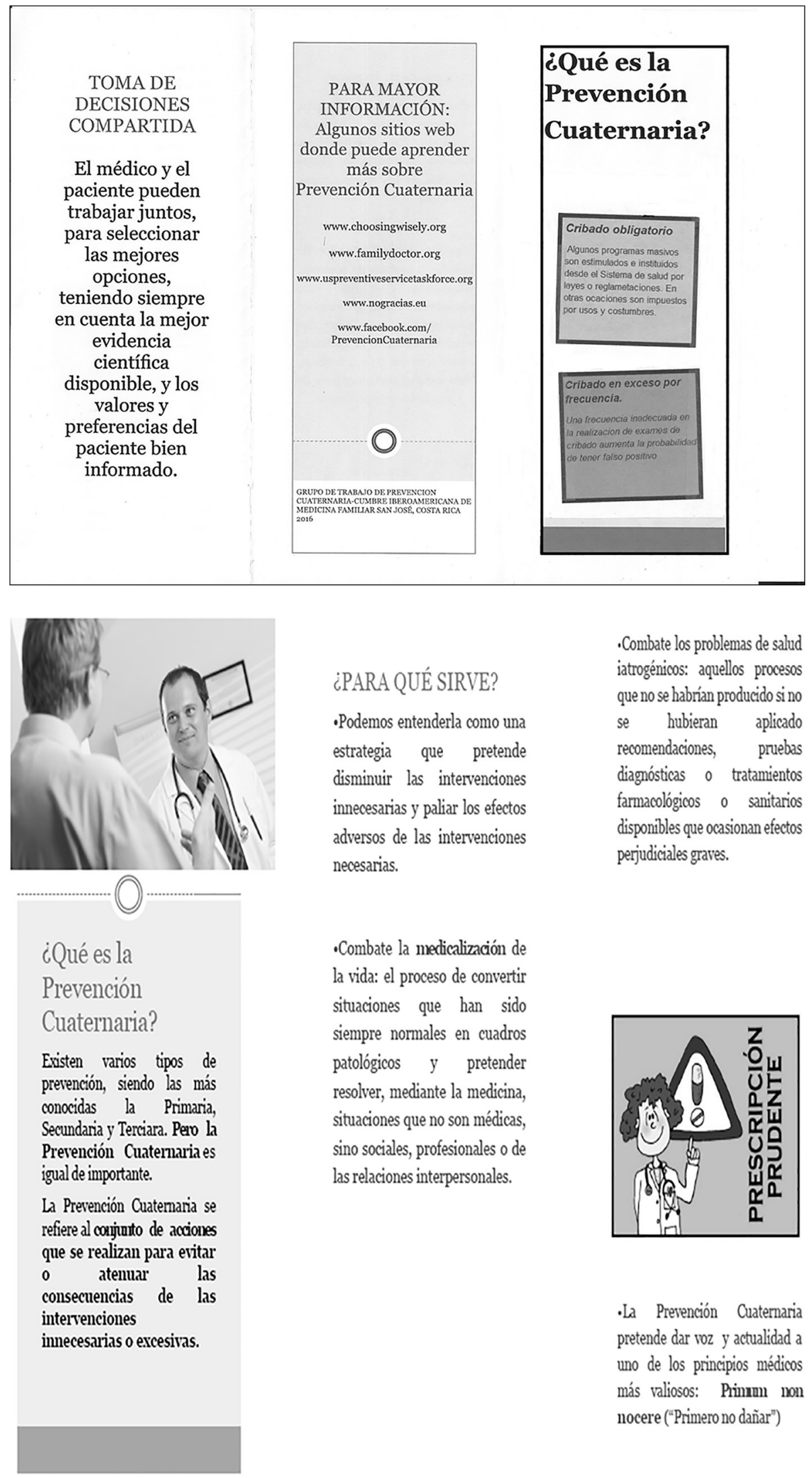

\section{¿PARA QUÉ SIRVE?}

-Podemos entenderla como una estrategia que pretende disminuir las intervenciones innecesarias y paliar los efectos adversos de las intervenciones necesarias.

-Combate la medicalización de la vida: el proceso de convertir situaciones que han sido siempre normales en cuadros patológicos y pretender resolver, mediante la medicina, situaciones que no son médicas, sino sociales, profesionales o de las relaciones interpersonales.
- Combate los problemas de salud iatrogénicos: aquellos procesos que no se habrían producido si no se hubieran aplicado recomendaciones, pruebas diagnósticas 0 tratamientos farmacológicos o sanitarios disponibles que ocasionan efectos perjudiciales graves.

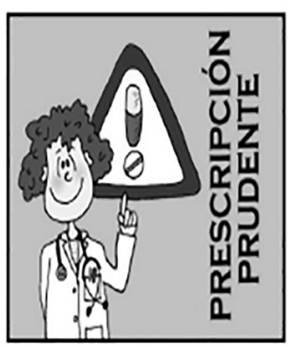

-La Prevención Cuaternaria pretende dar voz y actualidad a uno de los principios médicos más valiosos: Prinum non nocere ("Primero no dañar") 


\section{Referencias}

1. SUMEFAC/Grupo de Trabajo en Prevención Cuaternaria de CIMF. Acta de creación del grupo de trabajo. Sugerencias de la Primera reunión presencial del Grupo de Trabajo de CIMF a sociedades miembro [base de datos en Internet]*. Uruguay: SUMEFAC - Pizzanelli M.; 14 julio 2016, [actualizada julio 2016; acceso 14 de julio de 2016]. Disponible en: https://drive. google.com/file/d/OB2tjwHv1enqDZThrN2FDYzZpM3c/view?usp=sharing

2. Bentzen N, editor. Wonca International Dictionary of General/Family Practice: Wonca International Classification Committee; 1999.

3. Jamoulle M. A propos d'un dessin. Ethica Clinica [Internet]. 2013; 70:28-31. Disponible en: http://www.ph3c.org/PH3C/ docs/27/000281/0000416.pdf.

4. Leavell H, Clark E. Preventive Medicine for the Doctor in His Community an Epidemiologic Approach. McGraw-Hill, 1958.

5. Hart JT. The inverse care law. Lancet. 1971;1:405-412.

6. Illich I. Némesis Médica. Editorial Joaquín Mortiz, S.A. México D.F. La expropiación de la salud, 1978.

7. Nye RA. The evolution of the concept of medicalization in the late twentieth century. J Hist Behav Sci. 2003;39(2):115-129.

8. Jamoulle M. Quaternary prevention, an answer of family doctors to overmedicalization. Int J Health Policy Manag. 2015;4(2):61-64.

9. Cardoso RV. Medicalização e o cuidado em saúde na estratégia de saúde da família [thesis online]. Campinas: Universidade Estadual de Campinas; 2014. 268 p. Disponible en: http://www.ph3c.org/PH3C/docs/27/000281/0000605.pdf.

10. Jamoulle M, Bernstein J, Pizzanelli M, Silva AD, Wagner H. Quaternary prevention: a concept based on the time and the relationship. Second part. The surprising international dissemination of the concept. Médecine [Internet]. 2014 Feb 1 [cited 2015 Jul 19];10(2):75-7. Available from: http://www.jle.com/en/revues/med/e-docs/prevention_quaternaire_un_concept_fonde_sur_ le_temps_et_la_relation._deuxieme_partie._I_etonnante_diffusion_internationale_du_concept_299639/article.phtml?tab=texte

11. Socarrás Suárez MM, Bolet Astoviza M, Lara Rodríguez RF. Vínculo universidad-sociedad a través de la percepción de médicos y pacientes diabéticos sobre su enfermedad. Educ Med Super [Internet]. 2010 Sep [citado 2016 Jul 23];24(3):290-299. Disponible en: http://scielo.sld.cu/scielo.php?script=sci_arttext\&pid=S0864-21412010000300002\&lng=es.

12. López ML. Extensión universitaria y salud pública en Argentina como caso testigo en la región. Revista de Investigación Educativa, 34(1), 119-132. DOI: http://dx.doi.org/10.6018/rie.34.1.215411

13. Abreu Suárez G, González Valdés JA, Muñoz Peña R. La preparación del estudiante de Medicina para la eliminación de la tuberculosis. Educ Med Super [Internet]. 2013 Mar [citado 2016 Jul 23];27(1):38-45. Disponible en: http://scielo.sld.cu/scielo. php?script=sci_arttext\&pid=S0864-21412013000100006\&lng=es.

14. Vicente Sánchez B, Vicente Peña E, Altuna Delgado A, Identificación de individuos con riesgo de desarrollar diabetes tipo 2: una explicación necesaria. Rev. Finlay [Internet]. 2015 Sep [citado 2016 Jul 23];5(3):148-160. Disponible en: http://scielo.sld. cu/scielo.php?script=sci_arttext\&pid=S2221-24342015000300002\&lng=es.

15. Manterola Carlos, Otzen Tamara. Análisis Crítico de la Literatura Biomédica. Int. J. Morphol. [Internet]. 2014 Jun [citado 2016 Jul 23];32(2):599-607. Disponible en: http://www.scielo.cl/scielo.php?script=sci_arttext\&pid=S0717-95022014000200037\&lng=es.

16. Brodersen J, Schwartz LM, Woloshin S. Overdiagnosis: how cancer screening can turn indolent pathology into illness. APMIS. 2014 Aug;122(8):683-9

17. Sackett DL. The arrogance of preventive medicine. CMAJ [Internet]. 2002 8-20 [cited 2015 Sep 23];167(4):363-4. Available from:http://www.cmaj.ca/content/167/4/363

18. Welch HG. Should I be tested for cancer? University of California Press, 2004

19. United Nations, Sustainable Development Goals [sede Web]. New York: Division for Sustainable Development. Department of Economic and Social Affairs; julio 2016. [acceso 12 de julio de 2006]. Disponible en: https://sustainabledevelopment.un.org/

20. La Valle R. Prevención cuaternaria o la Medicina sin corbata. Archivos de Medicina Familiar y General 2015; 12(2): 5-6

21. Biodiversidadla.org. Carta de Brasilia: La salud es un derecho [sede Web]. Organización y movimientos de América; 3 de diciembre de 2015. [acceso 20 de julio de 2006]. Disponible en: http://www.biodiversidadla.org/Portada_Principal/ Documentos/Carta_de_Brasilia_La_salud_es_un_derecho

22. Menéndez, E. El Modelo Médico y la Salud de los Trabajadores. Salud Colectiva, La Plata, 2005.1 (1) 9-32.

23. World Health Organization [sede Web]*. WHO; octubre 2006 [acceso 20 de julio de 2006]. Constitución de la Organización Mundial de la Salud [1-20]. http://www.who.int/governance/eb/who_constitution_sp.pdf.

24. Jamoulle M. Quaternary prevention, an answer of family doctors to overmedicalization. Int J Health Policy Manag. 2015;4(2):61-64 